\title{
The Hypnotic Actions of the Fatty Acid Amide, Oleamide
}

\author{
Wallace B. Mendelson, M.D., and Anthony S. Basile, Ph.D.
}

Oleamide is an endogenous fatty acid amide which can be synthesized de novo in the mammalian nervous system, and has been detected in human plasma. It accumulates in the CSF of rats after six hours of sleep deprivation and induces sleep in naive rats and mice. Inhibition of the primary catabolic enzyme of oleamide (fatty acid amide hydrolase) by trifluoromethyl-octadecenone reduces sleep latency and increases total sleep time when given centrally to rats and peripherally to mice. While the mechanism of action of oleamide is unclear, it has been demonstrated to increase the amplitude of currents gated by 5-HT2a, 5HT2c and GABAa receptors. Moreover, the action of oleamide most relevant to sleep induction involves, in part, cannabinergic pathways, as evidenced by the ability of the cannabinoid antagonist SR 141716 to inhibit the hypnotic actions of $O A$. Nonetheless, enhancement of cannabinergic function may not be the only mechanism by which $O A$ alters sleep, as it can act synergistically with subthreshold doses of triazolam $(0.125 \mu \mathrm{g})$ to reduce sleep latency. These findings raise the possibility that $O A$ may be representative of a group of compounds which might be developed into clinically-used hypnotics, and are discussed in the context of fatty acid derivatives as modulators of neuronal function. [Neuropsychopharmacology 25:S36-S39, 2001] (C) 2001 American College of Neuropsychopharmacology. Published by Elsevier Science Inc.

\section{KEY WORDS: Oleamide; Fatty acids; Sleep; Hypnotics}

This review will describe the pharmacology of the unsaturated fatty acid amide oleamide (OA), presenting data indicating that this substance is formed in the mammalian CNS and has significant sleep-inducing properties. In addition, potential mechanisms by which OA induces sleep will be addressed, including interactions with serotonergic and GABAergic neurotransmission, with a particular emphasis on cannabinergic systems. Finally, the implications of these findings for understanding the function of endogenous sleep-promoting substances will be considered.

From the Sleep Research Laboratory, The University of Chicago, Chicago, IL (WBM), and Neuroscience Group, Laboratory of BioOrganic Chemistry, NIDDK, National Institutes of Health, Bethesda, MD (ASB)

Address correspondence to: Dr. Wallace Mendelson, Dept. of Psychiatry, The University of Chicago, 5841 S. Maryland Ave., MC 3077, Chicago, Illinois 60637 USA. Tel: (773) 834-0742, FAX: (773) 834-0744, e-mail: wmendels@yoda.bsd.uchicago.edu

\section{PHARMACOLOGY OF OLEAMIDE}

There has been longstanding interest in long chain, polyunsaturated free fatty acids as modulators of neurotransmitter and voltage-gated ion channel activity (Meves 1994). The physiologic significance of these compounds is unclear, however, because the concentrations required to affect receptor function are in the range of $10^{-5}$ to $10^{-4} \mathrm{M}$ (Witt and Nielsen 1994). In contrast, the related family of unsaturated fatty acids have similar abilities to modulate neurotransmitter gated ion channels, but with much higher potencies $\left(10^{-9}\right.$ to $10^{-7}$ M) (Devane et al. 1992; Huidobro-Toro and Harris 1996; Yost et al. 1998). Included in these compounds is oleamide (cis-9,10-octadecenamide), which is structurally related to sphingosine and sphinganine. OA, under its former name "cerebrodiene", was detected in the CSF of sleep deprived cats (Lerner et al. 1994) and was later reported to suppress motor activity, while inducing the sedated behavior and eye closing suggestive of sleep in rats (Cravatt et al. 1995). Subsequently, the absolute 
concentration of OA was found to increase more than 3fold in the CSF of rats subjected to six hours or more of sleep deprivation. Administration of OA was also found to reduce motor activity in the open field, while inducing electroencephalographic indices of sleep (Basile et al. 1999).

Oleamide is found endogenously; it can be formed de novo in brain microsomes (Sugiura et al. 1996) and is detectable in human luteal phase plasma (Arafat et al. 1989). Fatty acid amide hydrolase, its major catabolic enzyme, has been isolated and localized in a number of brain regions (Thomas et al. 1997). OA does not directly affect ligand binding to serotonin and GABA receptors (Basile et al. 1999; Huidobro-Toro and Harris 1996). However, OA increases currents gated by $5-\mathrm{HT}_{2 \mathrm{a}}$ and $5-\mathrm{HT}_{2 \mathrm{c}}$ receptors at concentrations of $200 \mathrm{nM}$ (HuidobroToro and Harris 1996) and it modulates GABA-induced chloride current amplitudes biphasically, enhancing currents in the hundreds of nanomolar range, but suppressing them at higher concentrations (Yost et al. 1998). Although OA does not appear to directly interact with cannabinoid receptors (Basile et al., 1999; Mechoulam et al., 1997), it raises concentrations of the endogenous CB-1 agonist anandamide (itself one of the family of unsaturated fatty acid amides) in vitro, possibly by interfering with hydrolysis (Mechoulam et al. 1997) or its cellular uptake (Hauer et al. 1998). Oleamide reportedly has little or no effect at muscarinic cholinergic, metabotropic glutamate, N-methyl-D-aspartate (NMDA), or $\alpha$-amino3-hydroxy-5-methyl-4-isoxozolepropionic acid (AMPA) receptors (Huidobro-Toro and Harris 1996). The mechanism by which OA induces these receptor effects is uncertain, though based on the experience with long chain, polyunsaturated free fatty acids (Meves 1994), it activates protein kinases, which phosphorylate consensus sites on receptor-related ion channels, thus altering their function. Alternatively, some of the effects induced by OA may result from the liberation of ammonia during its catabolism (Cravatt et al. 1995; Ha and Basile 1996).

\section{OLEAMIDE AS A SLEEP- INDUCING SUBSTANCE}

Studies in rats indicate that oleamide enhances sleep (Table 1). Microinjection of 2.8 and $5.6 \mu \mathrm{g}$ intraventricularly during daytime recordings significantly shortens EEG sleep latency (Basile et al. 1999). Presumably because of its very rapid metabolism, no significant effect is seen on total sleep in rats under these conditions. In mice, $10 \mathrm{mg} / \mathrm{kg}$ given intraperitoneally significantly shortens sleep latency and increases total sleep time, primarily by increasing NONREM sleep, with no significant effect on REM (Mendelson and Basile 2000a).

When trifluoromethyl-octadecenone (LOH-1-151),
Table 1. Effects of Oleamide IP on Sleep in Mice $(n=6)$

\begin{tabular}{llcc}
\hline Sleep measure & Treatment & Value (min) & $p<$ \\
\hline Sleep onset latency & Vehicle & $17.3 \pm 2.1$ & \\
& OA 10 mg/kg & $6.6 \pm 1.2$ & .01 \\
Total sleep time (min) & Vehicle & $64.0 \pm 4.1$ & \\
& OA 10 mg/kg & $82.6 \pm 3.9$ & .01 \\
REM sleep time (min) & Vehicle & $3.9 \pm 1.4$ & \\
& OA 10 mg/kg & $3.6 \pm 1.6$ & NS \\
nonREM sleep time (min) & Vehicle & $60.2 \pm 3.7$ & \\
& OA 10 mg/kg & $78.7 \pm 4.2$ & .01 \\
Wake time after sleep onset & Vehicle & $35.9 \pm 2.4$ & \\
& OA 10 mg/kg & $31.0 \pm 3.4$ & NS \\
REM sleep onset latency & Vehicle & $64.7 \pm 9.2$ & \\
& OA 10 mg/kg & $66.7 \pm 18.8$ & NS \\
& & &
\end{tabular}

Values represent mean \pm SEM minutes. NS $=$ not statistically significant. Data are derived from two hour recordings in 6 male C57 BL-6 mice following administration of $\mathrm{OA}$ at 10:00 AM, in a 12:12 L:D condition such that lights come on at 8:00 AM.

an inhibitor of the major degradative enzyme for OA (fatty acyl amide hydrolase, type I) is administered intraventricularly to rats, however, sleep latency is reduced, and total sleep is increased (Mendelson et al. 2000c). A similar effect is seen when LOH-1-151 in a dose of $10 \mathrm{mg} / \mathrm{kg}$ is given intraperitoneally to mice, as seen in Figure 1 and Figure 2.

\section{OLEAMIDE GIVEN IN COMBINATION WITH RECEPTOR BLOCKERS}

One way to explore which receptors might mediate the sleep-inducing effects of oleamide would of course be to observe sleep after administering it in combination with blockers for the receptors it is known to affect in

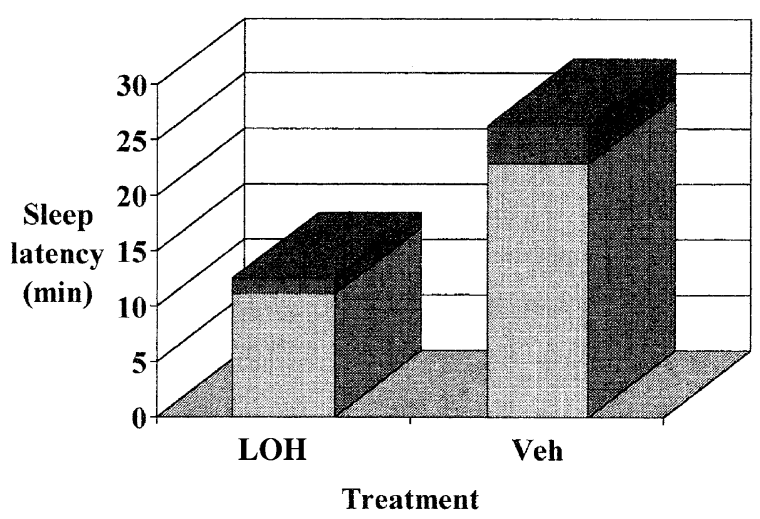

Figure 1. Effects of trifluoromethyl-octadecenone (LOH-1151) $10 \mathrm{mg} / \mathrm{kg}$ IP on sleep latency in mice. Values represent mean \pm SEM minutes. Effect of treatment was significant $(p<$ $.02)$ by 2 -tailed $t$-test. Data derived from a study in which 2-hour recordings were performed after injection at 10:00 A.M., under L:D conditions such that lights come on at 8:00 A.M. 


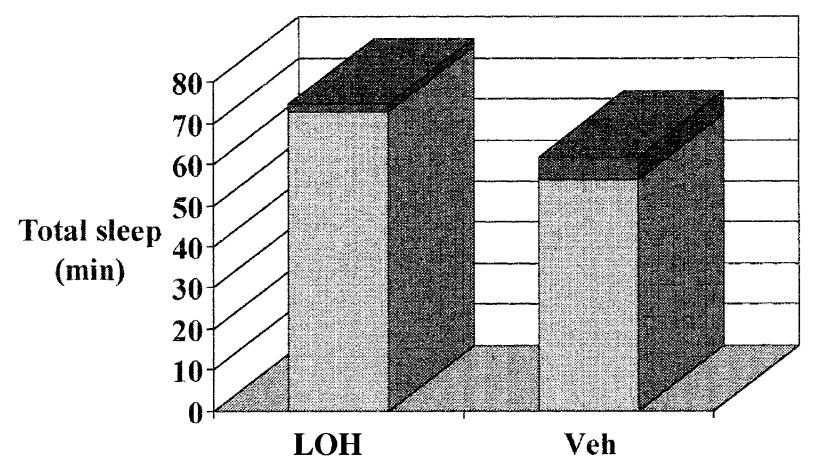

Treatment

Figure 2. Effects of trifluoromethyl-octadecenone (LOH1-151) $10 \mathrm{mg} / \mathrm{kg}$ IP on total sleep time in mice. Values represent mean \pm SEM minutes. Effect of treatment was significant $(p<.05)$ by 2 -tailed $t$-test. Treatment conditions are the same as in Figure 1.

vitro. One such agent is SR-141716, which selectively blocks the CB1 receptor with a $\mathrm{K}_{\mathrm{i}} \sim 5.6 \mathrm{nM}$, and which prevents such cannabinoid actions as memory disruption, analgesia and hypothermia (Terranova et al. 1996). Although higher peripheral doses may increase wakefulness (Santucci et al. 1996), low intraventricular doses $(3 \mu \mathrm{g})$ were determined to have no effect on sleep in rats following daytime injections. When given in combination with $2.8 \mu \mathrm{g}$ OA, SR-141716 was found to prevent the significant reduction in sleep latency observed when OA was given by itself (Mendelson and Basile 1999).

To this author's knowledge, OA has not yet been given in combination with a benzodiazepine recognition site antagonist. When a dose of OA two orders of magnitude lower than those which induce sleep $(0.0175$ $\mu \mathrm{g})$ is given intraventricularly to rats, along with a very low dose of triazolam $(0.125 \mu \mathrm{g})$, neither agent alone alters sleep, but the combination has a potent hypnotic action (Mendelson and Basile 2000b).

\section{CONCLUSIONS}

In summary, $\mathrm{OA}$ is a substance which accumulates in the CSF of sleep-deprived animals, and which induces sleep when administered to rats and mice. The observation that its hypnotic effects are prevented by a cannabinoid-1 receptor blocker suggests that one of its mechanisms of action involves an alteration (probably indirect) of CB-1 receptor function. The observation that it has a synergistic action with triazolam leaves open the possibility that its interaction with $\mathrm{GABA}_{\mathrm{A}}$ receptors has functional significance as well, or at least that they both affect a common downstream pathway. The possibility that OA may induce sleep via alteration of 5-HT receptors remains to be explored.
One implication of OA being a compound which is synthesized in the mammalian CNS and accumulates in the CSF during sleep deprivation, is of course that it is potentially a "hypnotoxin", an endogenous sleep regulatory substance. A number of authors have developed proposed sets of criteria for an endogenous sleep substance, e.g., that it should act at physiologic concentrations and that there is no evidence of tolerance. The challenge which awaits the proponents of any one potential sleep inducing factor is the large number of such substances (at last count, 30-40). If future studies lead to making a case for $\mathrm{OA}$ as such a substance, one of the intriguing aspects of $\mathrm{OA}$ is that it modulates at least three major receptor systems that affect sleep (5-HT, $\mathrm{GABA}_{\mathrm{A}}$ and CB-1). It appears that it does not do so by directly altering ligand binding, for at concentrations up to $50 \mu \mathrm{M}$, it had no effect on the binding of $\left[{ }^{3} \mathrm{H}\right]$ muscimol, $\left[{ }^{3} \mathrm{H}\right]$ flunitrazepam or $\left[{ }^{3} \mathrm{H}\right]$ ketanserin to $\mathrm{GABA}_{\mathrm{A}}$, benzodiazepine or $5-\mathrm{HT}_{2}$ receptors, respectively, as determined in rat brain membrane preparations (Basile et al. 1999). Alternatively, one possible mechanism by which it might do this is by activating protein kinases and hence changing the phosphorylation state of a variety of receptors. The $\mathrm{GABA}_{\mathrm{A}}$ receptor subunits are characterized by various consensus phosphorylation sites for a number of kinases (Leidenheimer et al. 1991). It is possible, for instance, that a receptor system, let us say the $\mathrm{GABA}_{\mathrm{A}}$ complex, might respond to GABA differently in the phosphorylated versus the dephosphorylated state. Protein phosphorylation at a tyrosine kinase-dependent site as well as at a distinct but uncharacterized site that is dephosphorylated by calcineurin have been reported to be necessary for $\mathrm{GABA}_{\mathrm{A}}$ receptor function (Huang and Dillon 1998). Insofar as OA alters a range of receptor systems, it is possible that it might have a more fundamental organizing role in the regulation of sleep and waking.

In addition to offering a potential insight into physiologic sleep regulation, $\mathrm{OA}$ is of interest for at least three clinical reasons. The identification of an agent this potent in inducing sleep raises the possibility, of course, that it might be the prototype of a new form of clinical hypnotic medication. Second, oleamide is widely used as an industrial lubricant in polyolefin manufacturing, and is found in many types of food containers (Cooper et al. 1995). Finally, there has been growing interest in the role of the related family of omega-3 polyunsaturated fatty acids in normal and abnormal CNS function, including a possible relationship to human affective disorders (Hebbeln and Salem 1999; Peet et al. 1998; Maes and Smith 1998). One ongoing hypothesis is that an interaction of these compounds with depressive symptomatology may involve their effects on 5-HT receptors. Interestingly, the amides of these compounds have only $40-60 \%$ as much activity as oleamide (Bolger et al. 1998). Thus the possibility remains open that an 
understanding of the interactions of oleamide with 5-HT receptors may be useful in future investigations of depression or the development of antidepressants.

\section{ACKNOWLEDGMENTS}

This work was partially supported by NIH grants 2PO1 AG 11412-03 and K07 HL03640.

\section{REFERENCES}

Arafat ES, Trimble JW, Andersen RN, Dass C, Desiderio DM (1989): Identification of fatty acid amides in human plasma. Life Sci 45:1679-1687

Basile A, Hanus L, Mendelson WB (1999): Characterization of the hypnotic properties of oleamide. NeuroReport 10:947-951

Bolger DL, Patterson JE, Jin Q (1998): Structural requirements for 5-HT2a and 5-HT1a serotonin receptor potentiation by the biologically active lipid oleamide. PNAS 95:4102-4107

Cooper I, Lord T, Tice PA (1995): Hydrolysis studies on oleamide in simulated gastrointestinal fluids. Food Addit Contam 12:769-777

Cravatt BF, Prospero-Garcia O, Siuzdak G, Gilula NB, Henriksen SJ, Boger DL, Lerner RA (1995): Chemical characterization of a family of brain lipids that induce sleep. Science 268:1506-1508

Devane WA, Hanus L, Breuer A, Pertwee RG, Stevenson LA, Griffin G, Gibson D, Mandelbaum A, Etinger A, Mechoulam R (1992): Isolation and structure of a brain constituent that binds to the cannabinoid receptor [see comments]. Science 258:1946-1949

Ha J-H, Basile AS (1996): Modulation of ligand binding to components of the GABAA receptor complex by ammonia: implications for the pathogenesis of hyperammonemic syndromes. Brain Res 720:35-44

Hauer KL, Bakhshan F, Lin H, Pidgeon C, Barker EL (1998): The sleep inducing lipid oleamide can potentiate the endogenous cannabinoid anandamide by transport inhibition. ACNP Annual Meeting, Las Colinas, Puerto Rico Abstract: 150

Hebbeln JR, Salem N (1999): Dietary polyunsaturated fats and depression: when cholesterol does not satisfy. Am J Clin Nutr 61:1-9

Huang R-Q, Dillon GH (1998): Maintenance of recombinant type A g-aminobutyric acid receptor function: role of protein tyrosine phosphorylation and calcineurin. JPET 286:243-255

Huidobro-Toro JP, Harris RA (1996): Brain lipids that induce sleep are novel modulators of 5-hydroxytryptamine receptors. Proc Natl Acad Sci 93:8078-8082

Leidenheimer NJ, Browning MD, Harris RA (1991): GABAa receptor phosphorylation: multiple sites, actions and artifacts. TIPS 12:84-87

Lerner RA, Siuzdak G, Prospero-Garcia O, Henriksen SJ, Boger DL, Cravatt BF (1994): Cerebrodiene: A brain lipid isolated from sleep deprived cats. Proc Natl Acad Sci USA 91:9505-9508

Maes M, Smith RS (1998): Fatty acids, cytokines and major depression. Biol Psychiatry 43:313-314

Mechoulam R, Fride E, Hanus L, Sheskin T, Di Marzo V, Bayewitch M, Vogel Z (1997): Anandamide may mediate sleep induction [letter]. Nature 389:25-26

Mendelson WB, Basile AS (1999): The hypnotic actions of oleamide are blocked by a cannabinoid receptor antagonist. NeuroReport 10:3237-3239

Mendelson WB, Basile AS (2000a) Sleep-inducing effects of oleamide in mice. 15th Congress of European Sleep Research Society Istanbul, September. 12-16

Mendelson WB, Basile AS (2000b): Synergistic interaction of the hypnotic effects of oleamide and triazolam. Sleep 23 (suppl 2):A162

Mendelson WB, Hanus L, Basile AS (2000c): The hypnotic effects of LOH-1-151, an inhibitor of oleamide catabolism. Sleep 23(suppl 2):A163

Meves H (1994): Modulation of ion channels by arachidonic acid. Prog Neurobiol 43:175-186

Peet M, Murhphy B, Shay J, Horrobin D (1998): Depletion of omega-3 fatty acid levels in red blood cell membranes of depressive patients. Biol Psychiat 43:315319

Santucci V, Storme JJ, Soubrie P (1996): Arousal-enhancing properties of the CB1 cannabinoid receptor antagonist SR 141716 A in rats as assessed by electroencephalographic spectral and sleep-waking cycle analysis. Life Sci 58:PL103-PL110

Sugiura T, Kondo S, Kodaka T (1996): Enzymatic symthesis of oleamide (cis-9, 10-octadecenoamide), an endogenous sleep-inducing lipid, by rat brain microsomes. Biochem Mol Biol Int 40:931-938

Terranova J-P, Storme J-J, Lafon N, Perio A, Rinaldi-Carmina M, Le Fur G, Soubrie P (1996): Improvement of memory in rodents by the selective CB1 cannabinoid receptor antagonist, SR 141716. Psychopharmacology 126:165-172

Thomas EA, Carson MJ, Neal MJ, Sutcliffe JG (1997): Unique allosteric regulation of 5-hydroxytryptamine receptormediated signal transduction by oleamide. Proc Natl Acad Sci USA 94:14115-14119

Witt M-R, Nielsen M (1994): Characterization of the influence of unsaturated free fatty acids on brain GABAbenzodiazepine receptor binding in vitro. J Neurochem 62:1432-1439

Yost CS, Leonoudakis BS, Koblin DD, Bornheim LM, Hampson AJ, Gray AT (1998): Oleic amide potentiates benzodiazepine-sensitive GABAa receptor activity but does not alter MAC. Anaesthesia and Analgesia 86:12941300 